\title{
Novel monoclonal antibodies to normal and pathologically altered human TDP-43 proteins
}

\author{
Linda K Kwong ${ }^{1}$, David J Irwin ${ }^{1,2}$, Adam K Walker ${ }^{1}$, Yan Xu', Dawn M Riddle', John Q Trojanowski ${ }^{1}$ \\ and Virginia M Y Lee ${ }^{1 *}$
}

\begin{abstract}
The RNA/DNA-binding protein, TDP-43, is the key component of ubiquitinated inclusions characteristic of amyotrophic lateral sclerosis (ALS) and the majority of frontotemporal lobar degeneration (FTLD-TDP) referred to collectively as TDP-43 proteinopathies. To further elucidate mechanisms of pathological TDP-43 processing and identify TDP-43 epitopes that could be useful as potential biomarkers of TDP-43 proteinopathies, we developed a panel of novel monoclonal antibodies (MAbs) directed at regions extending across the length of TDP-43. Here, we confirm previous observations that there is no or minimal accumulation of TDP-43 N-terminal domains in neocortical inclusions in human TDP-43 proteinopathy tissues and we identify a subset of these MAbs that are specific for human versus mouse TDP-43. Notably, one of these MAbs recognized an epitope that preferentially detected pathological TDP-43 inclusions with negligible reactivity for normal nuclear TDP-43 resembling anti-phospho-TDP-43 specific antibodies that only bind pathological TDP-43. Hence, we infer that this new MAb recognizes a phosphorylation independent but disease-specific pathologic conformation in abnormal TDP-43. These data suggest that the novel MAbs reported here will be useful for patient-oriented research as well as for studies of animal and cell-based models of TDP-43 proteinopathies including ALS and FTLD-TDP.
\end{abstract}

Keywords: TDP-43, Monoclonal antibody characterization, FTLD-TDP, ALS, Biomarker

\section{Introduction}

Since the landmark discovery of the RNA/DNA binding protein, TDP-43, as the main constituent of ubiquitinpositive, tau/synuclein-negative inclusions in amyotrophic lateral sclerosis (ALS) and frontotemporal lobar degeneration (FTLD) referred to as FTLD-TDP [1], there have been numerous advances in our understanding of these seemingly disparate clinical and neuropathological syndromes (reviewed in [2]). Indeed, ALS and FTLD-TDP are now considered to be different manifestations along a continuum or spectrum of TDP-43 proteinopathies [3]. Further, hierarchical non-random progression of TDP-43 pathology in ALS [4] and FTLD-TDP [5] suggest potential neuron-to-neuron spread of TDP-43 as a major driving force in the progression of the neurodegenerative process. Indeed, cell culture experiments suggest the possibility

\footnotetext{
* Correspondence: vmylee@upenn.edu

${ }^{1}$ Center for Neurodegenerative Disease Research \& Institute on Aging, Department of Pathology and Laboratory Medicine, Perelman School of Medicine, University of Pennsylvania, Philadelphia, PA 19104-4283, USA Full list of author information is available at the end of the article
}

of transmission of pathological TDP-43 between cells [6] similar to those reported in animal and cellular models of tauopathies $[7,8]$ and Parkinson's disease [9-12]. Accordingly, strategies to prevent or slow the transmission of pathogenic forms of TDP-43 in affected patients with passive immune therapy using disease specific monoclonal antibodies (MAbs) to pathological TDP-43 could emerge as a major therapeutic approach for treatment of FTLDTDP and ALS. To that end and for future research on animal- and cell-based models of TDP-43 proteinopathies, well-characterized MAbs to normal and pathological epitopes in TDP-43 are urgently needed. Here we describe the generation and characterization of novel MAbs that detect normal and disease-specific epitopes spanning the length of TDP-43.

\section{Materials and methods}

\section{Recombinant TDP-43 production}

Human recombinant TDP-43 (rTDP-43) proteins including full length TDP-43 (FL-rTDP-43), i.e. amino acids (aa) 1-414, N-terminally truncated TDP-43 (Nt-rTDP-43), aa 
1-261, and C-terminally truncated TDP-43 (Ct-rTDP-43), aa 182-414 were expressed in BL21 (DE3) E. coli cells using the pCOLD vector system (Takara Bio Inc., Japan). TDP-43 proteins were extracted from E. coli $16 \mathrm{~h}$ after IPTG induction, and the vast majority of the rTDP-43 proteins were present in inclusion bodies. The bacterial pellet was sequentially extracted with PBS and 1\% Triton X-100 to remove soluble bacterial proteins. For immunization, the Triton X-100 insoluble rTDP-43 was solublized with $1 \%$ Sarkosyl buffer or $4 \mathrm{M}$ urea. Sarkosyl was removed by exhaustive dialysis before use. For epitope mapping, the Triton X-100 insoluble rTDP-43 was solublized directly in Laemmli sample buffer, and immunoblot (IB) analysis was done as described previously [13,14].

\section{MAb generation and screening}

Murine MAbs were raised against human rTDP-43 proteins using similar methods described previously [15-18]. Mice were immunized with FL-rTDP-43 or Nt-rTDP-43. Briefly, rTDP-43 proteins were emulsified with Freund's adjuvant and injected subcutaneously into BALB/c mice. The mice were boosted 3 additional times at 2 week intervals. Three days prior to harvesting spleens for fusion to generate MAbs, the mice received intraperitoneal injection of rTDP-43 without adjuvant. Splenic lymphocytes were fused to Sp2 mouse myeloma cells using the polyethylene glycol method to produce mouse hybridomas as described [15]. Animal care and all procedures performed here were conducted in accordance with the NIH Guide for the Care and Use of Experimental Animals and approved by the University of Pennsylvania Institutional Animal Care and Use Committee. Hybridoma supernatants were initially screened for the ability to recognize human FL-rTDP-43 by indirect enzyme-linked immunosorbant assay (ELISA), and positive clones were further evaluated by IB using FL-rTDP-43 and by immunohistochemistry (IHC) on formalin and ethanol fixed paraffin-embedded sections of FTLD-TDP cortex (see below). Cells from positive hybridomas were expanded and re-screened as above.

\section{Epitope mapping and characterization of MAbs}

The TDP-43 domains harboring the epitopes recognized by these new MAbs were first estimated using FL-rTDP43, Nt-rTDP-43 and Ct-rTDP-43 in IB studies similar to our characterization of MAbs we previously generated to tau and alpha-synuclein $[18,19]$. After probing with the MAbs, the bound antibodies were detected with horseradish peroxidase-conjugated goat anti-mouse IgG (Jackson ImmunoResearch, West Grove, PA). The blots were visualized using the chemiluminescent system as described previously [1]. For more refined mapping of the TDP-43 protein domains harboring the epitopes detected by these MAbs, indirect ELISAs were performed using peptides corresponding to different regions spanning the length of TDP-43: aa 6-24, 40-54, 110-147, 175-187, 189-195, 203-220, 235-243, 251-263, 287-322, 341-352, and 394-414. Cross-reactivity to mouse TDP-43 was evaluated by IB using RIPA solubilized human and mouse cell lysates [20] and by IHC using paraffin-embedded mouse and human brain sections (see below). To determine human specificity, the immunoreactivity of each MAb to human versus mouse TDP-43 was compared as described by Giasson et al. [19]. Similarly, immunocytochemistry (ICC) was performed using QBI293 and Neuro2a cells to verify cross-reactivity [20]. Human cortical urea extracts from a normal control and a FTLD-TDP case were used to determine if the MAbs recognized the pathological signature of TDP-43 by IB [1]. Isotypes of the MAbs were determined using a commercial rapid ELISA mouse antibody kit (Thermo Fisher Scientific, Rockford, IL, USA).

\section{Sandwich ELISA}

The 384-well format sandwich ELISA method used to evaluate the MAbs was similar to those described previously for a 96-well format, except the sample volumes used were reduced to $30 \mu \mathrm{L} /$ well [21]. Briefly, plates were coated with $5 \mu \mathrm{g} / \mathrm{mL}$ of capture antibody (rabbit polyclonal Ct or Nt TDP-43 (pAb) or MAbs) and incubated overnight at $4{ }^{\circ} \mathrm{C}$. Plates were blocked for a minimum of 3 days at $4^{\circ} \mathrm{C}$ with Block-Ace (AbD Serotec, Raleigh, NC). After the blocking solution was removed, 0,1 or $10 \mathrm{ng} / \mathrm{mL}$ of FL-rTDP-43 were added to wells and incubated for $16 \mathrm{~h}$ at $4^{\circ} \mathrm{C}$. Plates were subsequently washed and incubated with $0.2,1$ or $10 \mu \mathrm{g} / \mathrm{mL}$, detection antibody (MAbs for the rabbit $\mathrm{pAb}$ coated plates and rabbit $\mathrm{pAb}$ for MAb coated plates) for $16 \mathrm{~h}$ at $4^{\circ} \mathrm{C}$. The plates were then washed and incubated with HRP-conjugated reporting antibodies (goat anti-mouse or anti-rabbit, respectively), for $1 \mathrm{~h}$ at $25^{\circ} \mathrm{C}$ (Santa Cruz Biotechnology). A tetramethyl benzidine peroxidase substrate system was used for the color development step (Thermo Fisher Scientific, Rockford, IL, USA). A serial diluted rTDP-43 standard curve, $10 \mathrm{ng} / \mathrm{mL}$ to $1 \mathrm{pg} / \mathrm{mL}$, was used to estimate the level of detergent soluble TDP-43 in brain tissue or cell extracts. Samples were done in quadruplicates. Detection limit is defined as average optical density of reagent background (no rTDP43 added) plus 4 times the standard deviation.

\section{IHC studies}

Human CNS tissues from the superior temporal cortex (FTLD-TDP subtypes A-D) and spinal cord (ALS) (one patient each) were examined to evaluate the reactivity of TDP-43 MAbs in pathological aggregations and neuronal nuclei (for demographic details please see Additional file 1: Table S1). Neuropathological examination of human cases was performed as described previously [22] using current neuropathological criteria for the diagnosis of 
FTLD-TDP and ALS $[23,24]$. The brain and spinal cord tissues were obtained at autopsy and processed following standard established protocol [22]. Autopsy and required informed consent were performed in accordance with the University of Pennsylvania Institutional Review Board. Paraffin-embedded human CNS tissue sections were deparaffinized in xylenes and rehydrated in a descending series of ethanol. Endogenous peroxidases were blocked with $5 \%$ hydrogen peroxide in methanol, and sections were microwaved in 1\% antigen unmasking solution (Vector Laboratories, Burlingame, CA) at $99^{\circ} \mathrm{C}$ for $15 \mathrm{~min}$, blocked in $2 \%$ fetal bovine serum in $0.1 \mathrm{M}$ Tris buffer and incubated with TDP-43 MAbs in ascites or as purified IgG. Affinity-purification of IgG was done using the HiTrap Protein G column according to manufacturer instructions (GE Healthcare, Piscataway, NJ). MAbs were incubated overnight at $4^{\circ} \mathrm{C}$ at concentrations of $1: 1 \mathrm{~K}-1: 100 \mathrm{~K}$ (ascites) or $0.03-1.0 \mu \mathrm{g} / \mathrm{ml}$ (purified IgG). Reactivity of novel TDP-43 MAbs was compared with a previously characterized disease-specific MAb to TDP-43 phosphorylated at serine 409 and 410 (MAb p409/410) [25] at concentrations of 1:250-1:500.

IHC of mouse CNS tissues was performed in the same manner described above, and in addition, we used a mouse on mouse (MOM) immunodetection kit (Vector Laboratories) to minimize endogenous mouse IgG background. For all sections, labeling was detected using species-specific biotinylated secondary antibodies incubated for $1 \mathrm{~h}$ and the Vectastain $A B C$ kit followed by the ImmPACT ${ }^{\mathrm{mm}} \mathrm{DAB}$ Substrate kit (Vector Laboratories). Slides were coverslipped with CytoSeal 60 mounting medium (Thermo Scientific).

The degree of non-pathological nuclear TDP-43 and pathological TDP-43 deposits in human lower motor neurons (LMN), white-matter glial cell inclusions (GCI), neuronal cytoplasmic inclusions (NCI), dystrophic neurites (DN) and neuronal nuclear inclusions (NII) were reviewed by a trained examiner (DJI) and rated on a semiquantitative scale $(0=$ none, $r=$ rare,$+=$ few,$++=$ moderate, $+++=$ many) for each MAb. Digital images of IHC results were obtained using an Olympus BX 51 microscope equipped with a bright-field and fluorescence light source with a DP-71 digital camera (Olympus; Center Valley, PA) and DP manager software (Olympus).

\section{Results}

\section{MAb generation and epitope mapping}

To gain further insights into the molecular and neuropathological roles of TDP-43 in neurodegeneration, we generated new MAbs for future studies of TDP-proteinopathies. Approximately 4500 potential MAb clones from three hybridoma fusion procedures were screened by FL-rTDP-43 indirect ELISA of which 168 clones were positive on this initial screen and all were evaluated further in IB and IHC studies. Only 45 clones continued to show immunoreactivity toward TDP-43 in the secondary screens and all of these MAbs were studied further to determine the epitopes and their antibody isotype as described $[18,19]$. Using FL-rTDP-43, Nt-rTDP-43, and Ct-rTDP-43 for IB analyses, the epitope region was estimated to be within aa 1-181 for 6 MAbs, within aa 182-261 for 5 MAbs, and within aa 262-414 for 34 MAbs (data not shown and Table 1). Indirect ELISA using a panel of TDP-43 peptides as antigens further mapped some of the epitopes to smaller regions of TDP43. Four of the MAbs recognized the aa 6-24 peptide, 7 recognized the aa 289-322 peptide and 3 recognized the extreme C-terminus peptide (aa 394-414) (Figure 1). After additional cell passages and/or subcloning procedures, 18 MAbs were selected for further characterization based on the hybridomas' apparent clonality and stability. These MAbs were shown to have their putative epitopes mapped to the regions in TDP-43 shown in Table 1 . Next, these MAbs were evaluated for their effective use in several assays including: immunoprecipitation and IB of human cell lysates, IHC, ICC, and sandwich ELISA. Most of the MAbs were found to have efficacy in all assays tested, except for MAb 211 (Table 1) which failed as a detection antibody by ELISA even at a 20 fold higher concentration than that used for other MAbs (data not shown).

To determine whether or not the selected 18 MAbs detected both human and mouse TDP-43, IHC was performed using normal human and non-transgenic mouse brain sections. All 18 MAbs detected nuclear TDP-43 in sections of human hippocampus (Figure 2a,c), but 7 of these MAbs did not detect mouse TDP-43 by IHC (compare Figure $2 \mathrm{~b}$ to Figure $2 \mathrm{~d}$ ). To determine if these 7 apparently human specific MAbs detect mouse TDP-43 in other applications, we compared their immunoreactivity toward human versus mouse TDP-43 from cultured cells on IB. Only 3 of these 7 MAbs (138, 5123, and 5104) did not detect the mouse protein. The remaining 4 MAbs, including MAbs 5032 and 5095, recognized mouse TDP43, albeit with lower efficiency than the human protein (Figure 2e). Thus, we identified 11 MAbs that detected both mouse and human TDP-43 with equal affinity, 4 that preferentially recognized human versus mouse TDP43 but were not completely human specific, and 3 that detected human but not mouse TDP-43 in the assays used here.

\section{ELISA studies}

As of now, there is no reliable method to quantify TDP-43 proteins in biofluids such as plasma or cerebrospinal fluid (CSF) samples which would be useful as a biomarker assay. In order to determine if the MAbs generated in this study might be useful for developing an assay to detect TDP-43 in CSF or plasma, we evaluated them in 
Table 1 Summary of the characterization of New TDP-43 MAbs

\begin{tabular}{|c|c|c|c|c|c|c|c|c|c|}
\hline MAb & Isotype & Epitope (aa) & IP & IB & IHC & ICC & ELISA capture & ELISA detect & Human preference \\
\hline 211 & $\lg G_{1}$ & $6-24$ & - & + & + & + & + & - & - \\
\hline 5031 & $\lg G_{2 a}$ & $25-181$ & + & + & + & + & + & + & - \\
\hline 138 & $\lg G_{2 a}$ & $182-261$ & + & + & + & + & + & + & + \\
\hline 5060 & $\lg G_{2 a}$ & $182-261$ & ND & + & + & + & + & + & - \\
\hline 5117 & $\lg G_{2 a}$ & $287-322$ & ND & + & + & + & + & + & - \\
\hline 5156 & $\operatorname{lgG}_{2 a}$ & $287-322$ & + & + & + & + & + & + & - \\
\hline 205 & $\lg G_{2 a}$ & $262-391$ & + & + & + & + & + & + & + \\
\hline 241 & $\lg G_{1}$ & 262-391 & + & + & + & + & + & + & + \\
\hline 5028 & $\lg G_{1}$ & $262-391$ & + & + & + & + & + & + & - \\
\hline 5032 & $\lg G_{1}$ & $262-391$ & + & + & + & + & + & + & + \\
\hline 5053 & $\lg G_{1}$ & $262-391$ & ND & + & + & - & + & + & - \\
\hline 5056 & $\lg G_{2 a}$ & 261-391 & + & + & + & + & + & + & - \\
\hline 5089 & $\lg G_{2 b}$ & 261-391 & + & + & + & + & + & + & - \\
\hline 5095 & $\lg G_{1}$ & $261-391$ & + & + & + & + & + & + & + \\
\hline 5104 & $\lg G_{1}$ & 261-391 & + & + & + & + & + & + & + \\
\hline 5123 & $\lg G_{1}$ & 261-391 & + & + & + & + & + & + & + \\
\hline 5101 & $\lg G_{1}$ & 261-391 & + & + & + & + & + & + & - \\
\hline 5195 & $\lg G_{2 a}$ & $394-414$ & + & + & + & + & + & + & - \\
\hline
\end{tabular}

This table summarizes the properties the new MAbs described here. New abbreviation: ND = not determined.

a sandwich ELISA using previously developed Nt- or Ct-TDP-43 pAbs [17] as capture or detection antibody. As mentioned above and shown in Table 1, 17 MAbs were capable of detecting and capturing FL-rTDP-43 by ELISA. This suggested that one or more of these MAbs may be advanced for the development of a sensitive ELISA to quantify TDP-43, especially in biofluids. This is exemplified by studies using MAb 205 for capture and the $\mathrm{Ct}$-pAb as the detection antibody in an ELISA that showed a lower limit of detection for human rTDP-43 of $\sim 25 \mathrm{pg} / \mathrm{mL}$ (see Additional file 1: Figure S1). Using this sensitive ELISA, we were able to quantify the amount of detergent soluble TDP-43 in QBI293 cell lysates and in human FTLD-TDP cortical grey matter lysates, $\sim 30 \mathrm{ng} / \mathrm{mg}$ protein and $\sim 850 \mathrm{ng} / \mathrm{g}$ grey, respectively.

\section{Immunoreactivity in human tissue}

TDP-43 MAbs were screened for reactivity in human CNS tissues using IHC in the spinal cord of ALS and superior temporal cortex of morphological subtypes of TDP-43 (Type A-D) [24]. Semi-quantitative analyses for each type of morphologic inclusion (i.e. LMN, NCI, DN, NII, and GCI) in ALS and FTLD-TDP are summarized in Table 2. MAbs 211 and 5031, which are specific for the $\mathrm{N}$-terminus of TDP-43 (i.e. regions A and B as illustrated in Figure 1) showed robust reactivity for normal nuclear TDP-43, but 5031 detected more pathology in the ALS spinal cord and FTLD-TDP temporal cortex than 211 (Figure 3a,c and data not shown). Indeed, the amount of pathologic TDP-43 in cortical sections detected by 5031 was modest, while 211 did not detect significant amounts

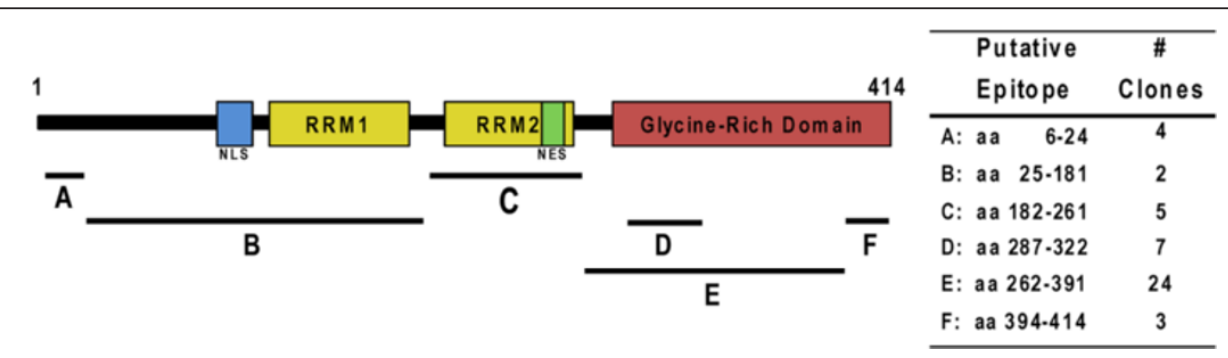

Figure 1 Schematic representation of TDP-43 domains containing epitopes recognized by the MABs reported here. The TDP-43 domains harboring the epitopes recognized by 45 newly generated MAbs were mapped to regions along the entire length of TDP-43 (A-F) by immunoblot and indirect peptide ELISA and the putative amino acid stretches included in these epitopes is depicted in the table to the right of the schematic. RRM: RNA-recognition motif; NLS: bipartite nuclear localization signal; NES: nuclear export signal. 


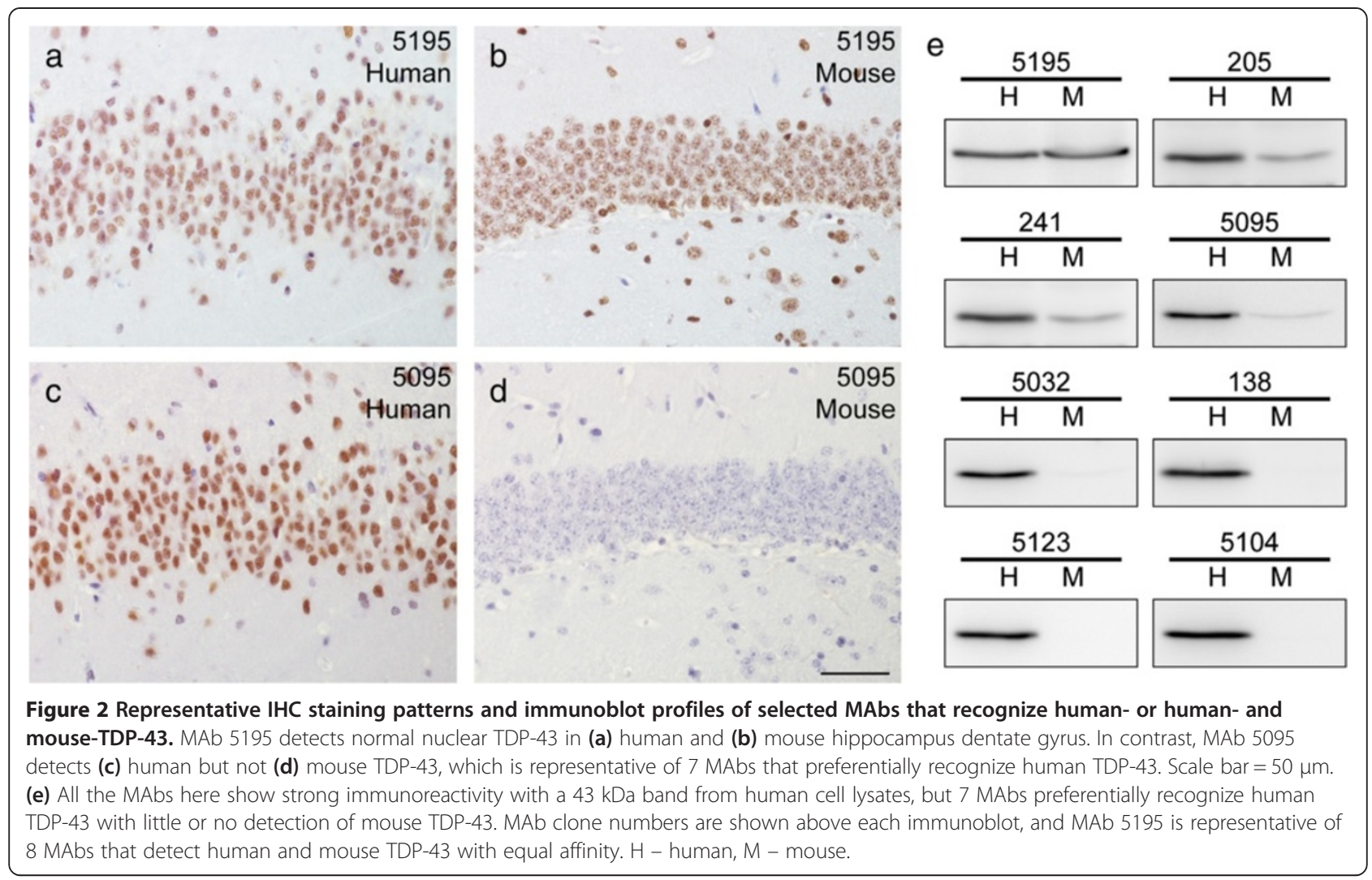

of neocortical TDP-43 deposits in the FTLD-TDP cases. Thus, the N-terminal specific MAbs preferentially detected TDP-43 pathology mainly in spinal cord motor neurons, which contains a predominance of full-length TDP-43 [13]. In contrast, MAbs specific for the RNA recognition motif 2 (RRM2), i.e. Region $\mathrm{C}$ in Figure 1 or the $\mathrm{C}$-terminal glycine rich domain (i.e. Regions D-F) were immunoreactive to all forms of neocortical pathological TDP-43 inclusions seen in FTLD-TDP subtypes, as well as LMN inclusions in ALS from a moderate to high degree for most inclusion types (Figure 3b,d). The minimal limited immunoreactivity of MAbs specific for $\mathrm{N}$-terminal regions in neocortical TDP-43 inclusions of FTLD-TDP cases was confirmed using IB analyses of human CNS urea extract of FTLD-TDP brains which showed that a C-terminal fragment (CTF) of TDP-43 was detected by MAbs specific for regions in the $\mathrm{C}$-terminus (Figure $3 \mathrm{f}$ ), but not the $\mathrm{N}$-terminus specific MAb 5031 (Figure 3e). Moreover, MAb 5031 also did not detect the pathological hyperphosphorylated $45 \mathrm{kD}$ band or the high molecular weight (MW) smears which were detected MAb 5056, consistent with previous findings [13].

The reactivity to neocortical TDP-43 pathology by MAbs specific for C-terminal TDP-43 epitopes was accompanied by a moderate to high reactivity for normal nuclear TDP-43 by all MAbs except MAb 138. MAb 138, which is specific for region $\mathrm{C}$ in the RRM2, displayed high reactivity to pathological TDP-43 deposits similar to other TDP-43 MAbs specific for Regions C-F, in the absence of significant normal nuclear TDP-43 staining (Figure 4). This preferential detection of pathological TDP43 inclusions with minimal or no normal nuclear TDP-43 staining by IHC is similar to the staining pattern seen with pathology-specific phospho-TDP-43 specific MAbs such as the p409/410 MAb mentioned above. There was no regional predilection for this pattern, as it was evident in both the neocortex and spinal cord of ALS and FTLD-TDP patients. To further test this observation we analyzed the hippocampal dentate gyrus from a nonneurodegenerative control case with MAb138 under a range of dilutions to establish if the negligible degree of normal nuclear TDP staining was reproducible in a control case. As shown in Online Resource 1 (Additional file 1: Figure S2), the pattern of immunoreactivity observed with MAb 138 did not appear to reflect antibody dilution or affinity. Finally, there were no clear differences between the ability of the different MAbs to detect the different morphological types of TDP-43 inclusions (i.e. DN, NCI, NII, GCI and granular cytoplasmic TDP deposits), FTLD-TDP subtypes A-D as classified by MacKenzie et al. [24] or clear effects of fixative method or post-mortem interval in this screening survey. 
Table 2 IHC properties of new TDP-43 MAbs

\begin{tabular}{|c|c|c|c|c|c|c|c|c|}
\hline \multirow{2}{*}{$\begin{array}{l}\text { Region/ } \\
\text { antibody }\end{array}$} & \multicolumn{3}{|c|}{ Spinal cord } & \multicolumn{5}{|c|}{ Temporal cortex } \\
\hline & Nuclei & LMN & $\overline{\mathrm{GCl}}$ & Nuclei & $\mathrm{NCl}$ & $\mathrm{DN}$ & NII & $\mathrm{GCl}$ \\
\hline \multicolumn{9}{|c|}{ A (aa 6-24) } \\
\hline 211 & +++ & + & + & +++ & 0 & 0 & 0 & 0 \\
\hline \multicolumn{9}{|c|}{ B (aa 25-181) } \\
\hline 5031 & ++ & +++ & + & ++ & r & + & + & $r$ \\
\hline \multicolumn{9}{|c|}{ C (aa 182-261) } \\
\hline 138 & 0 & +++ & + & 0 & +++ & ++ & + & + \\
\hline 5060 & +++ & +++ & + & ++ & + & + & ++ & $r$ \\
\hline \multicolumn{9}{|c|}{ D (aa 287-322) } \\
\hline 5117 & ++ & ++ & $r$ & +++ & +++ & ++ & r & ++ \\
\hline 5156 & +++ & +++ & r & +++ & +++ & +++ & + & ++ \\
\hline \multicolumn{9}{|c|}{ E (aa 262-391) } \\
\hline 205 & +++ & +++ & r & ++ & +++ & ++ & + & + \\
\hline 241 & ++ & +++ & + & ++ & +++ & ++ & + & + \\
\hline 5053 & +++ & +++ & + & +++ & +++ & +++ & ++ & + \\
\hline 5056 & ++ & ++ & $r$ & +++ & +++ & +++ & ++ & + \\
\hline 5095 & +++ & ++ & r & +++ & ++ & ++ & ++ & + \\
\hline \multicolumn{9}{|c|}{ F (aa 392-414) } \\
\hline 5195 & + & +++ & r & +++ & ++ & ++ & ++ & + \\
\hline
\end{tabular}

This table summarizes the IHC characteristics of the new MAbs described here. They are identified by the numbers in the first column under A-F. The aa numbers in parenthesis indicates the different protein domains within which the epitopes reside that are recognized by these new MAbs. New abbreviations: Nuclei = Normal endogenous TDP-43 staining; LMN = lower motor neuron cytoplasmic inclusions, $r=$ rare.

\section{Discussion}

Here we describe the generation and characterization of several novel TDP-43 specific MAbs that recognize epitopes distributed over the entire length of TDP-43 (Figure 1, Table 1) most of which are useful for immunoprecipitation and IB methods, as well as for use as capture or detection MAbs in an ELISA assay in addition to being effective in ICC and IHC studies. Notably, using MAb 205 in a sandwich ELISA as the capture antibody, we were able to detect human rTDP-43 at a lower limit of $25 \mathrm{pg} / \mathrm{ml}$ (Additional file 1: Figure S1). Moreover, we also identified a subset of 7 MAbs that preferentially detect human TDP-43 and not mouse TDP-43 while we showed that 11 of these new MAbs detect both human and mouse TDP-43 with equal robustness. Further, using these new MAbs, we confirm previous results [13] showing a predominance of CTF in neocortical TDP-43 inclusions of FTLD-TDP brains and full-length TDP-43 in the spinal cord of ALS spinal cord (Figure 3). Remarkably, we identified a unique MAb (138) that binds to an epitope in the RRM2 region that shows a preferential reactivity for pathological TDP-43 inclusions and minimal or no recognition of normal nuclear TDP-43, similar to disease specific phospho-TDP-43 specific MAbs such as the p409/410 MAb (Figure 4).

Since mouse TDP- 43 shares $96 \%$ identity with the human protein, it is not uncommon for antibodies made against human TDP-43 to react with the mouse protein. Surprisingly, in this study we identified 7 MAbs that preferentially recognize human TDP-43, 6 of which bind to an epitope in regions downstream of aa 261. These MAbs are all human specific on IHC, but have varying immunoreactivity on IB to mouse TDP-43, from none detected to $\sim 30 \%$ cross-reactivity. The varying reactivity of these novel MAbs to mouse TDP-43 most likely reflects underlying differences in aa sequence between human and mouse TDP-43. Thus, these novel and relatively humanspecific TDP-43 MAbs will be very useful for studies of transgenic animal models of TDP-43 proteinopathies, including differentiating mouse versus human TDP-43 CTF that accumulate in pathologic inclusions.

Our IHC studies in human ALS and FTLD-TDP tissues with these novel MAbs suggests they may provide insights into the pathophysiology of TDP-43 misfolding and aggregation in human disease. First, our results reinforce previous findings [13] of a predominance of CTF in neocortical inclusions in FTLD-TDP, confirmed by minimal reactivity of our novel N-terminus specific MAbs (i.e. regions A-B) with brain TDP-43 pathology. Interestingly, MAb 211, which is specific for the extreme $\mathrm{N}$-terminus (Region A), did not detect significant neocortical pathology while MAb 5031 (Region B) detected rare to low levels of neocortical pathology (Figure 3). Furthermore, MAb 5031 did not appear to favor the detection of a particular cell type (i.e. oligodendrocyte, astrocyte, neurons) or cellular inclusions type (i.e. NCI, DN, etc.) suggesting the lack of alternative processing of pathological TDP-43 within different cell types in the neocortex of FTLD-TDP brains. However, the cause of preferential accumulation of full-length TDP-43 into LMN neuron and glial inclusions in the spinal cord of ALS remains unclear.

IB analysis of human brain urea extracts also showed that the Nt MAbs had limited immunoreactivity for the pathological biochemical features of FTLD-TDP. MAb 5031 detected the $43 \mathrm{kD}$ band with similar sensitivity as the Ct MAb 5056; however, MAb 5031 failed to detect the $\mathrm{Ct}$ fragment, as well as the $45 \mathrm{kD}$ band and the high MW smear. This finding is consistent with our previously developed Nt-pAb, which had much less immunoreactivity toward the pathological TDP-43 on IB when compared to the Ct-pAb [13]. It is possible that conformational changes in pathological TDP-43 render the Nt epitope inaccessible to the antibody by immunoblotting. However, we cannot rule out the possibility that the majority of protein species in the $45 \mathrm{kD}$ band and the high $\mathrm{MW}$ smear might not be full length TDP-43. 


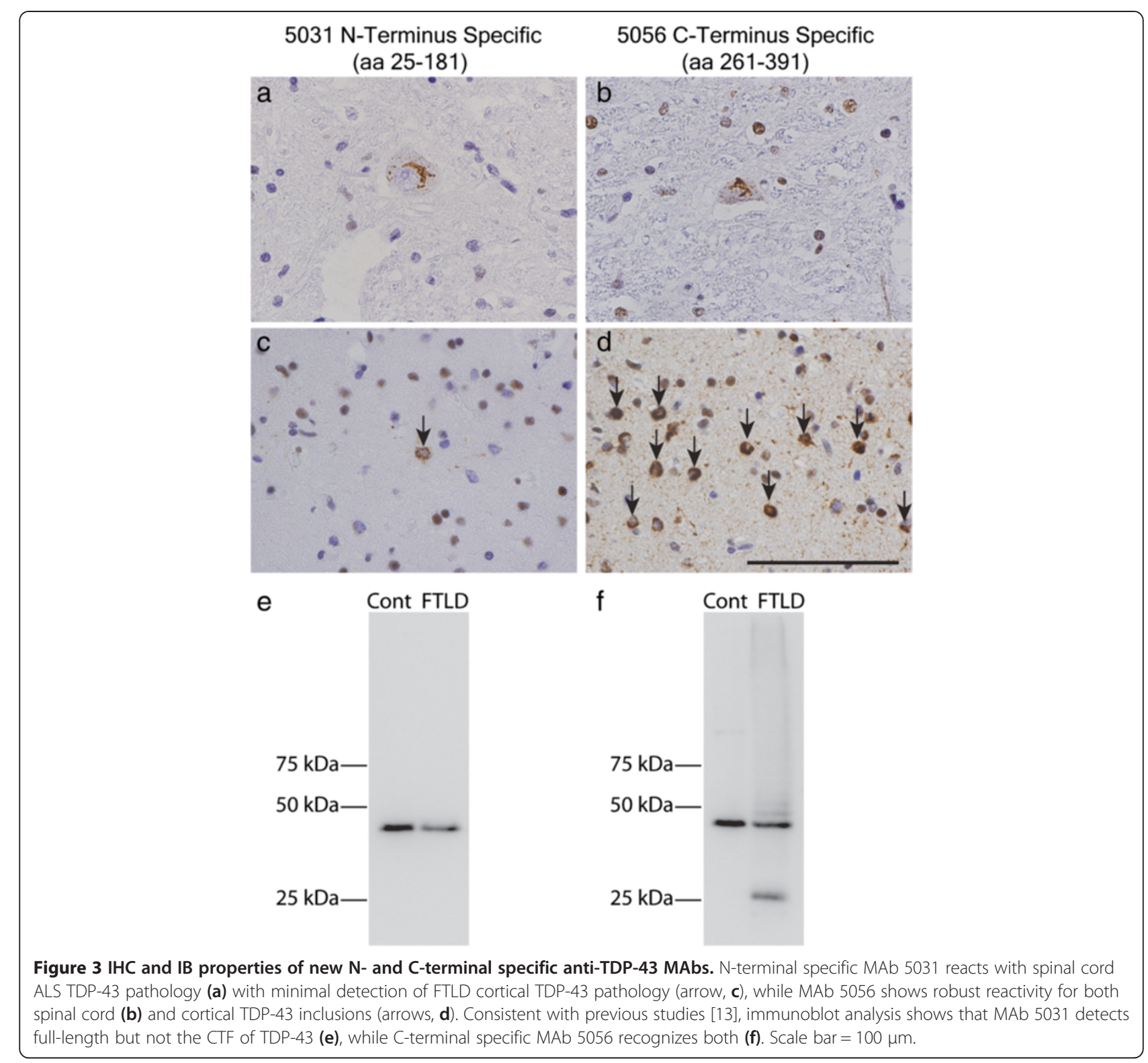

MAbs specific for presumed sequences from aa 182 and beyond towards the C-terminus of TDP-43 (i.e. Regions C-F) showed robust reactivity for neocortical TDP-43 pathology across FTLD-TDP subtypes and ALS spinal cord inclusions. These MAbs directed against epitopes in the mid- and C-terminus of TDP-43 detected a moderate to abundant normal nuclear TDP-43 with the exception of MAb 138 (Region C) (Figure 4). MAb 138 has a higher affinity for pathological TDP-43 similar to the pathology-specific MAb p409/410. MAb 5060 was also mapped to Region $C$, but did not share this unique IHC characteristic with MAb 138. It is unlikely that the 2 MAbs have identical epitope within Region $\mathrm{C}$, which spans 79 aa. Thus, further epitope mapping is needed to determine their specific epitopes within this region and clarify the discordant nature of the IHC reactivity between these MAbs.

There are several possible explanations for the unique IHC staining by MAb 138. First, there is a disease-specific pathological modification to one or more aa within the epitope recognized by MAb 138, but this seems unlikely as all these novel MAbs were generated using un-modified human rTDP-43. Second, a recent report suggests that the RRM2 region can undergo self-association when interacting with nucleic acids and this could alter the conformation of TDP-43 or the accessibility of the epitope recognized by MAb 138 [26]. Finally, it is possible that under physiological conditions the MAb 138 specific epitope may be buried or bound to nucleic acids and not be accessible to the antibody when present normally 


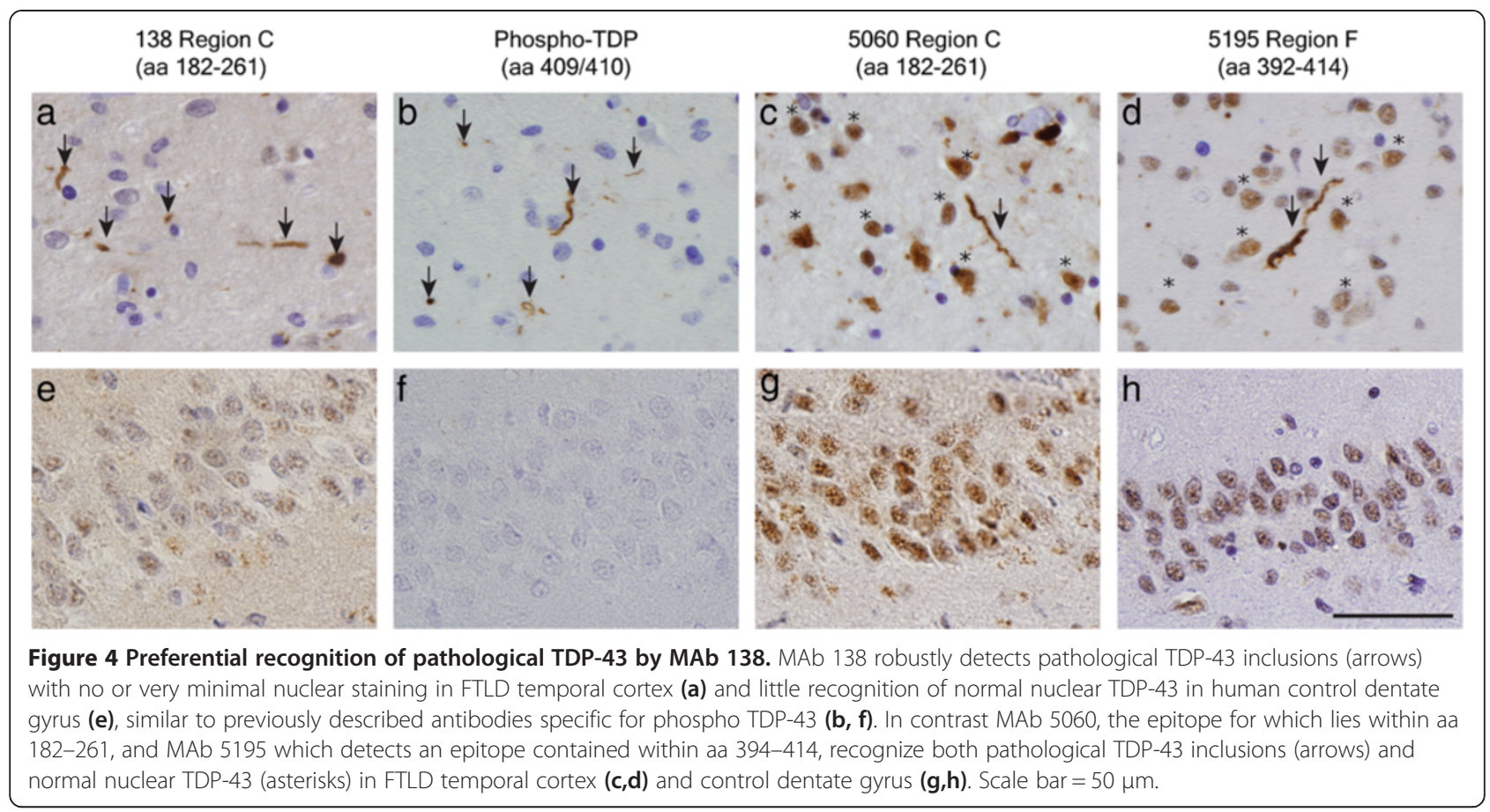

in the nucleus while becoming more accessible in TDP-43 inclusions. Of note, MAb 138 does detect the normal nuclear TDP-43 in QBI293 cells by ICC (Table 2), albeit to a much less degree or intensity than the other MAbs reported in our study (data not shown) and most published pan-TDP-43 antibodies. Our previous findings that TDP43 could be extracted biochemically with low-stringency buffers from cultured cells [27], but was only extractable with high-stringency detergent-containing buffer from CNS tissue [1], supports a potential structural difference between nuclear TDP-43 in CNS and cultured cells. Thus, the slight immunoreactivity of MAb 138 with non-pathological TDP43 in non-neuronal cell culture is difficult to interpret precisely at this time, but we will continue to characterize the basis for its preferential recognition of pathological TDP-43 in a large comprehensive neuropathological survey and further neuronal culture experiments.

Protein misfolding and prion-like spread of neurodegenerative disease proteins has emerged in the forefront of research for drug-development in FTLD-TDP and ALS [28,29]. Although TDP-43 transgenic mouse models with cytoplasmic TDP-43 accumulations that recapitulate clinical and pathological phenotypes of the human disease remain to be developed, novel MAbs such as those reported here will be critical to evaluate the sequential deposition of pathological TDP-43 for pre-clinical evaluations of novel therapeutics focused on TDP-43 aggregation as well as for use as potential immune therapies. Finally, there is an urgent need for FTLD-TDP/ALS-specific biomarkers [30], and ELISAs that are able to reliably measure CSF and/or plasma levels of TDP-43 will be useful not only for diagnosis, but also for monitoring responses to disease modifying therapies for FTLD-TDP, ALS and other TDP43 proteinopathies [31]. Hence, we expect that the panel of novel MAbs described here will be informative tools for future patient oriented and experimental studies of TDP-43 proteinopathies.

\section{Additional file}

Additional file 1: Figure S1. Representative ELISA results for a newly developed MAb, Figure S2. Serial Dilutions of MAb 138 show minimal nuclear reactivity and Table S1. Summary of patient demographics.

\section{Competing interests}

The authors declare that there are no competing interests to disclose.

\section{Authors' contributions}

LKK, DJ, AKW, VML, and JQT designed the study and wrote the manuscript. DMR carried out the hybridoma fusion and subcloning as well as the ICC experiments. LKK, AKW, and YX performed the MAb characterizations and evaluations. DI levaluated the human neuropathology. All authors read and approved the final manuscript.

\section{Acknowledgements}

This study was supported by NIH grants AG032953, AG-17586, AG-10124, and the Koller Foundation for ALS Research. DJI is supported by the Penn Institution for Translational Medicine and Therapeutics (ITMAT) and AKW is supported by the Australian National Health \& Medical Research Council C.J. Martin Biomedical Early Career Fellowship (1036835). We also would like to thank the patients and families for their meaningful contribution that made this work possible.

\section{Author details}

${ }^{1}$ Center for Neurodegenerative Disease Research \& Institute on Aging, Department of Pathology and Laboratory Medicine, Perelman School of Medicine, University of Pennsylvania, Philadelphia, PA 19104-4283, USA. ${ }^{2}$ Penn Frontotemporal Degeneration Center, Department of Neurology Perelman School of Medicine, University of Pennsylvania, Philadelphia, PA 19104-6021, USA. 
Received: 10 March 2014 Accepted: 10 March 2014

Published: 31 March 2014

\section{References}

1. Neumann M, Sampathu DM, Kwong LK, Truax AC, Micsenyi MC, Chou TT, Bruce J, Schuck T, Grossman M, Clark CM, McCluskey LF, Miller BL, Masliah E, Mackenzie IR, Feldman H, Feiden W, Kretzschmar HA, Trojanowski JQ, Lee VM (2006) Ubiquitinated TDP-43 in frontotemporal lobar degeneration and amyotrophic lateral sclerosis. Science 314(5796):130-133, doi:10.1126/science.1134108

2. Lee EB, Lee VM, Trojanowski JQ (2012) Gains or losses: molecular mechanisms of TDP43-mediated neurodegeneration. Nat Rev Neurosci 13(1):38-50, doi:10.1038/nrn3121

3. Geser F, Martinez-Lage M, Robinson J, Uryu K, Neumann M, Brandmeir NJ, Xie SX, Kwong LK, Elman L, McCluskey L, Clark CM, Malunda J, Miller BL, Zimmerman EA, Qian J, Van Deerlin V, Grossman M, Lee VM, Trojanowski JQ (2009) Clinical and pathological continuum of multisystem TDP-43 proteinopathies. Arch Neurol 66(2):180-189, doi:10.1001/archneurol.2008.558

4. Brettschneider J, Del Tredici K, Toledo JB, Robinson JL, Irwin DJ, Grossman M, Suh E, Van Deerlin VM, Wood EM, Baek Y, Kwong L, Lee EB, Elman L, McCluskey L, Fang L, Feldengut S, Ludolph AC, Lee VM, Braak H, Trojanowski JQ (2013) Stages of pTDP-43 pathology in amyotrophic lateral sclerosis. Ann Neurol 74(1):20-38, doi:10.1002/ana.23937

5. Brettschneider J, Del Tredici K, Irwin DJ, Grossman M, Robinson JL, Toledo JB, Fang L, Van Deerlin VM, Ludolph AC, Lee VM, Braak H, Trojanowski JQ (2014) Sequential distribution of pTDP-43 pathology in behavioral variant frontotemporal dementia (bvFTD). Acta Neuropathol 127(3):423-439, doi:10.1007/s00401-013-1238-y

6. Furukawa Y, Kaneko K, Watanabe S, Yamanaka K, Nukina N (2011) A seeding reaction recapitulates intracellular formation of Sarkosyl-insoluble transactivation response element (TAR) DNA-binding protein-43 inclusions. J Biol Chem 286 (21):18664-18672, doi:10.1074/jbc.M111.231209

7. Guo JL, Lee VM (2013) Neurofibrillary tangle-like tau pathology induced by synthetic tau fibrils in primary neurons over-expressing mutant tau. FEBS Lett 587(6):717-723, doi:10.1016/j.febslet.2013.01.051

8. Iba M, Guo JL, McBride JD, Zhang B, Trojanowski JQ, Lee VM (2013) Synthetic tau fibrils mediate transmission of neurofibrillary tangles in a transgenic mouse model of Alzheimer's-like tauopathy. J Neurosci 33 (3):1024-1037, doi:10.1523/JNEUROSCI.2642-12.2013

9. Luk KC, Song C, O'Brien P, Stieber A, Branch JR, Brunden KR, Trojanowski JQ, Lee VM (2009) Exogenous alpha-synuclein fibrils seed the formation of Lewy body-like intracellular inclusions in cultured cells. Proc Natl Acad Sci U S A 106(47):20051-20056, doi:10.1073/pnas.0908005106

10. Volpicelli-Daley LA, Luk KC, Patel TP, Tanik SA, Riddle DM, Stieber A, Meaney DF, Trojanowski JQ, Lee VM (2011) Exogenous alpha-synuclein fibrils induce Lewy body pathology leading to synaptic dysfunction and neuron death. Neuron 72(1):57-71, doi:10.1016/j.neuron.2011.08.033

11. Luk KC, Kehm VM, Zhang B, O'Brien P, Trojanowski JQ, Lee VM (2012) Intracerebral inoculation of pathological alpha-synuclein initiates a rapidly progressive neurodegenerative alpha-synucleinopathy in mice. J Exp Med 209(5):975-986, doi:10.1084/jem.20112457

12. Luk KC, Kehm V, Carroll J, Zhang B, O'Brien P, Trojanowski JQ, Lee VM (2012) Pathological alpha-synuclein transmission initiates Parkinson-like neurodegeneration in nontransgenic mice. Science 338(6109):949-953, doi:10.1126/ science.1227157

13. Igaz LM, Kwong LK, Xu Y, Truax AC, Uryu K, Neumann M, Clark CM, Elman LB, Miller BL, Grossman M, McCluskey LF, Trojanowski JQ, Lee VM (2008) Enrichment of C-terminal fragments in TAR DNA-binding protein-43 cytoplasmic inclusions in brain but not in spinal cord of frontotemporal lobar degeneration and amyotrophic lateral sclerosis. Am J Pathol 173(1):182-194, doi:10.2353/ajpath.2008.080003

14. Igaz LM, Kwong LK, Chen-Plotkin A, Winton MJ, Unger TL, Xu Y, Neumann M, Trojanowski JQ, Lee VM (2009) Expression of TDP-43 C-terminal fragments in vitro recapitulates pathological features of TDP-43 proteinopathies. J Biol Chem 284(13):8516-8524, doi:10.1074/jbc.M809462200

15. Lee V, Wu HL, Schlaepfer WW (1982) Monoclonal antibodies recognize individual neurofilament triplet proteins. Proc Natl Acad Sci U S A 79(19):6089-6092

16. Lee VM, Page CD, Wu HL, Schlaepfer WW (1984) Monoclonal antibodies to gel-excised glial filament protein and their reactivities with other intermediate filament proteins. J Neurochem 42(1):25-32

17. Sampathu DM, Neumann M, Kwong LK, Chou TT, Micsenyi M, Truax A, Bruce J, Grossman M, Trojanowski JQ, Lee VM (2006) Pathological heterogeneity of frontotemporal lobar degeneration with ubiquitin-positive inclusions delineated by ubiquitin immunohistochemistry and novel monoclonal antibodies. Am J Pathol 169(4):1343-1352, doi:10.2353/ajpath.2006.060438

18. Kosik KS, Orecchio LD, Binder L, Trojanowski JQ, Lee VM, Lee G (1988) Epitopes that span the tau molecule are shared with paired helical filaments. Neuron 1(9):817-825

19. Giasson BI, Jakes R, Goedert M, Duda JE, Leight S, Trojanowski JQ, Lee VM (2000) A panel of epitope-specific antibodies detects protein domains distributed throughout human alpha-synuclein in Lewy bodies of Parkinson's disease. J Neurosci Res 59(4):528-533

20. Winton MJ, Igaz LM, Wong MM, Kwong LK, Trojanowski JQ, Lee VM (2008) Disturbance of nuclear and cytoplasmic TAR DNA-binding protein (TDP-43) induces disease-like redistribution, sequestration, and aggregate formation. J Biol Chem 283(19):13302-13309, doi:10.1074/jbc.M800342200

21. Skovronsky DM, Wang J, Lee VM, Doms RW (2000) Quantifying abeta(1-40) and abeta (1-42) using sandwich-ELISA. Methods Mol Med 32:79-89, doi:10.1385/1-59259-195-7:79

22. Toledo JB, Van Deerlin VM, Lee EB, Suh E, Baek Y, Robinson JL, Xie SX, McBride J, Wood EM, Schuck T, Irwin DJ, Gross RG, Hurtig H, McCluskey L, Elman L, Karlawish J, Schellenberg G, Chen-Plotkin A, Wolk D, Grossman M Arnold SE, Shaw LM, Lee VM, Trojanowski JQ (2013) A platform for discovery: the University of Pennsylvania Integrated Neurodegenerative Disease Biobank. Alzheimers Dement: , doi:10.1016/j.jalz.2013.06.003

23. Mackenzie IR, Neumann M, Bigio EH, Cairns NJ, Alafuzoff I, Kril J, Kovacs GG, Ghetti B, Halliday G, Holm IE, Ince PG, Kamphorst W, Revesz T, Rozemuller AJ, Kumar-Singh S, Akiyama H, Baborie A, Spina S, Dickson DW, Trojanowski JQ, Mann DM (2010) Nomenclature and nosology for neuropathologic subtypes of frontotemporal lobar degeneration: an update. Acta Neuropathol 119(1):1-4, doi:10.1007/s00401-009-0612-2

24. Mackenzie IR, Neumann M, Baborie A, Sampathu DM, Du Plessis D, Jaros E, Perry RH, Trojanowski JQ, Mann DM, Lee VM (2011) A harmonized classification system for FTLD-TDP pathology. Acta Neuropathol 122(1):111-113, doi:10.1007/s00401-011-0845-8

25. Neumann M, Kwong LK, Lee EB, Kremmer E, Flatley A, Xu Y, Forman MS, Troost D, Kretzschmar HA, Trojanowski JQ, Lee VM (2009) Phosphorylation of $\$ 409 / 410$ of TDP-43 is a consistent feature in all sporadic and familial forms of TDP-43 proteinopathies. Acta Neuropathol 117(2):137-149, doi:10.1007/s00401-008-0477-9

26. Kuo PH, Doudeva LG, Wang YT, Shen CK, Yuan HS (2009) Structural insights into TDP-43 in nucleic-acid binding and domain interactions. Nucleic Acids Res 37(6):1799-1808, doi:10.1093/nar/gkp013

27. Pesiridis GS, Tripathy K, Tanik S, Trojanowski JQ, Lee VM (2011) A "two-hit" hypothesis for inclusion formation by carboxyl-terminal fragments of TDP-43 protein linked to RNA depletion and impaired microtubule-dependent transport. J Biol Chem 286(21):18845-18855, doi:10.1074/jbc.M111.231118

28. Polymenidou M, Cleveland DW (2012) Prion-like spread of protein aggregates in neurodegeneration. J Exp Med 209(5):889-893, doi:10.1084/jem.20120741

29. Boxer AL, Gold M, Huey E, Gao FB, Burton EA, Chow T, Kao A, Leavitt BR, Lamb B, Grether M, Knopman D, Cairns NJ, Mackenzie IR, Mitic L, Roberson ED, Van Kammen D, Cantillon M, Zahs K, Salloway S, Morris J, Tong G, Feldman H, Fillit H, Dickinson S, Khachaturian Z, Sutherland M, Farese R, Miller BL, Cummings J (2013) Frontotemporal degeneration, the next therapeutic frontier: molecules and animal models for frontotemporal degeneration drug development. Alzheimers Dement 9(2):176-188, doi:10.1016/j.jalz.2012.03.002

30. Hu WT, Trojanowski JQ, Shaw LM (2011) Biomarkers in frontotemporal lobar degenerations-progress and challenges. Prog Neurobiol 95(4):636-648, doi:10.1016/j.pneurobio.2011.04.012

31. Steinacker $P$, Hendrich $C$, Sperfeld AD, Jesse S, von Arnim CA, Lehnert S, Pabst A, Uttner I, Tumani H, Lee VM, Trojanowski JQ, Kretzschmar HA, Ludolph A, Neumann M, Otto M (2008) TDP-43 in cerebrospinal fluid of patients with frontotemporal lobar degeneration and amyotrophic lateral sclerosis. Arch Neurol 65(11):1481-1487, doi:10.1001/archneur.65.11.1481

\section{doi:10.1186/2051-5960-2-33}

Cite this article as: Kwong et al:: Novel monoclonal antibodies to normal and pathologically altered human TDP-43 proteins. Acta Neuropathologica Communications 2014 2:33. 